TRANSACTIONS OF THE

AMERICAN MATHEMATICAL SOCIETY

Volume 351, Number 1, January 1999, Pages 323-341

S 0002-9947(99)02028-0

\title{
OPERATOR VALUED WEIGHTS WITHOUT STRUCTURE THEORY
}

\author{
TONY FALCONE AND MASAMICHI TAKESAKI
}

\begin{abstract}
A result of Haagerup, generalizing a theorem of Takesaki, states the following:

If $\mathcal{N} \subset \mathcal{M}$ are von Neumann algebras, then there exists a faithful, normal and semi-finite (fns) operator valued weight $T: \mathcal{M}_{+} \rightarrow \widehat{\mathcal{N}_{+}}$if and only if there exist fns weights $\tilde{\varphi}$ on $\mathcal{M}$ and $\varphi$ on $\mathcal{N}$ satisfying $\sigma_{t}^{\varphi}(x)=\sigma_{t}^{\tilde{\varphi}}(x) \forall x \in \mathcal{N}, t \in \mathbb{R}$. In fact, $T$ can be chosen such that $\tilde{\varphi}=\varphi \circ T ; T$ is then uniquely determined by this condition.

We present a proof of the above which does not use any structure theory.
\end{abstract}

\section{INTRODUCTION}

Consider the following theorem of Haagerup:

Theorem. Let $\mathcal{N} \subset \mathcal{M}$ be von Neumann algebras. There exists a faithful, semifinite normal operator valued weight $T: \mathcal{M}_{+} \rightarrow \widehat{\mathcal{N}_{+}}$if and only if there exist faithful semi-finite normal weights $\tilde{\varphi}$ on $\mathcal{M}$ and $\varphi$ on $\mathcal{N}$ such that

$$
\sigma_{t}^{\varphi}(x)=\sigma_{t}^{\tilde{\varphi}}(x), \quad x \in \mathcal{N} .
$$

If this condition is satisfied, then $T$ can be chosen in such a way that $\tilde{\varphi}=\varphi \circ T$; moreover, $T$ is uniquely determined by this identity.

Haagerup's proof of the above depends upon the use of structure theory, i.e., the existence of a crossed product decomposition of a general von Neumann algebra [Haa1], [Haa2]. However, it is possible to demonstrate this without recourse to the crossed product. The method for showing this depends critically on a result of Connes-Masuda which provides a converse to the theorem purporting the existence of the Radon-Nikodým cocycle derivative. (Masuda's result does not use structure theory, unlike that of Connes.) This result implies that, given an invariance condition like that in the hypothesis, a weight on $\mathcal{N}$ induces one on $\mathcal{M}$. With the ability to associate weights on $\mathcal{N}$ to weights on $\mathcal{M}$, it is possible to construct an operator valued weight $T: \mathcal{M}_{+} \rightarrow \widehat{\mathcal{N}_{+}}$.

The desire to recast the proof of the above in a form which does not depend on any structure theory is motivated by the belief that it is a result which is fundamental in the theory of non-commutative integration. Specifically, application of an operator valued weight to a positive element of $\mathcal{M}$ (which results in an element in

Received by the editors January 30, 1997.

1991 Mathematics Subject Classification. Primary 46L50; Secondary 22D25.

This work is supported, in part, by NSF Grant DMS95-00882.

(C)1999 American Mathematical Society 
the extended positive cone of $\mathcal{N}$ ) can and should be thought of as "partial integration." The "space" over which we are integrating is not always explicit; however, just as we often think of von Neumann algebras as "non-commutative $L^{\infty}$-spaces," we sense the presence of an underlying measure space indirectly, by observing the interactions of function-like objects defined on this "space." Moreover, this point of view allows us to interpret the expression $\tilde{\varphi}=\varphi \circ T$ as a form of Fubini's theorem (or more precisely, Tonelli's theorem). Because we believe that structure theory should, in some sense, be a consequence of the results of integration theory, and not vice-versa, a proof which does not involve the crossed product is indicated.

It should also be noted that work in this area was done by Hirakawa [Hir]. The work presented herein addresses the problem from a different direction than he did, however, and therefore represents a new approach.

\section{Some Preliminaries}

In order to discuss the subject of operator valued weights, we begin with a review of some of the relevant terms and concepts. When studying weights, we consider the extended positive real numbers $\mathbb{R}_{+} \cup\{\infty\}$. To study "unbounded conditional expectations" (i.e., operator valued weights), we need to consider the "extended positive part" $\widehat{\mathcal{N}_{+}}$of the von Neumann subalgebra $\mathcal{N}$ of $\mathcal{M}$. We begin with the following:

Definition 1.1. For a von Neumann algebra $\mathcal{M}$, the extended positive cone $\widehat{\mathcal{M}_{+}}$ of $\mathcal{M}$ is the set of maps $m: \mathcal{M}_{*}^{+} \rightarrow[0, \infty]$ with the following properties:

(i) $m(\lambda \varphi)=\lambda m(\varphi), \quad \varphi \in \mathcal{M}_{*}^{+}, \quad \lambda \geq 0$,

(ii) $m(\varphi+\psi)=m(\varphi)+m(\psi), \quad \varphi, \psi \in \mathcal{M}_{*}^{+}$,

(iii) $m$ is lower semi-continuous.

Clearly, the positive part $\mathcal{M}_{+}$of $\mathcal{M}$ is a subset of $\widehat{\mathcal{M}}_{+}$. It is easy to see that $\widehat{\mathcal{M}_{+}}$is closed under addition, multiplication by non-negative scalars and increasing limits.

Example 1.2. Let $\{\mathcal{M}, \mathfrak{H}\}$ be a von Neumann algebra and $A$ a positive selfadjoint operator on $\mathfrak{H}$ affiliated with $\mathcal{M}$. Suppose that

$$
A=\int_{0}^{\infty} \lambda d e(\lambda)
$$

is the spectral decomposition of $A$. For each $\varphi \in \mathcal{M}_{*}^{+}$, put

$$
m_{A}(\varphi)=\int_{0}^{\infty} \lambda d \varphi(e(\lambda))
$$

Then $m_{A}$ satisfies the conditions (i), (ii) and (iii) of Definition 1.1. The last condition, the lower semi-continuity, follows from

$$
m_{A}(\varphi)=\sup _{n} \varphi\left(A_{n}\right) \quad \text { with } \quad A_{n}=\int_{0}^{n} \lambda d e(\lambda) \in \mathcal{M}_{+} .
$$

It now follows that

$$
m_{A}\left(\omega_{\xi}\right)=\int_{0}^{\infty} \lambda d(e(\lambda) \xi \mid \xi)= \begin{cases}\left\|A^{1 / 2} \xi\right\|^{2}, & \xi \in \mathcal{D}\left(A^{1 / 2}\right), \\ +\infty, & \xi \notin \mathcal{D}\left(A^{1 / 2}\right) .\end{cases}
$$

If $B$ is another positive self-adjoint operator on $\mathfrak{H}$ affiliated with $\mathcal{M}$, then the equality $m_{A}=m_{B}$ means precisely $A=B$. Hence the map $A \mapsto m_{A} \in \widehat{\mathcal{M}_{+}}$is 
injective. Thus, the set of positive self-adjoint operators affiliated with $\mathcal{M}$ can be identified with a subset of the extended positive cone $\widehat{\mathcal{M}_{+}}$.

Definition 1.3. For $m, n \in \widehat{\mathcal{M}}_{+}, \quad \lambda \geq 0$ and $a \in \mathcal{M}$, we define the following operations:

$$
\begin{aligned}
(\lambda m)(\varphi) & :=\lambda m(\varphi), \quad \varphi \in \mathcal{M}_{*}^{+}, \\
(m+n)(\varphi) & :=m(\varphi)+n(\varphi), \quad \varphi \in \mathcal{M}_{*}^{+}, \\
\left(a^{*} m a\right)(\varphi) & :=m\left(a \varphi a^{*}\right), \quad \varphi \in \mathcal{M}_{*}^{+} .
\end{aligned}
$$

We also note here that $\sup _{i} m_{i}$ of an increasing net in $\widehat{\mathcal{M}}_{+}$can be naturally defined.

Lemma 1.4. Let $\{\mathcal{M}, \mathfrak{H}\}$ be a von Neumann algebra. To each $m \in \widehat{\mathcal{M}}_{+}$, there corresponds uniquely a pair $\{A, \mathfrak{K}\}$ of a closed subspace $\mathfrak{K}$ of $\mathfrak{H}$ and a positive selfadjoint operator $A$ on $\mathfrak{K}$ such that

(i) $\mathfrak{K}$ is affiliated with $\mathcal{M}$, in the sense that the projection to $\mathfrak{K}$ belongs to $\mathcal{M}$, and $A$ is affiliated with $\mathcal{M}$;

$$
m\left(\omega_{\xi}\right)= \begin{cases}\left\|A^{1 / 2} \xi\right\|^{2}, & \xi \in \mathcal{D}\left(A^{1 / 2}\right), \\ +\infty, & \text { otherwise. }\end{cases}
$$

Here $\omega_{\xi}$ means, of course, the functional $x \in \mathcal{M} \mapsto(x \xi \mid \xi)$.

The proof of the above lemma, as well as the next theorem and its corollary, is standard, and is therefore omitted. (See, for instance, [Haa1].)

We say that an element $m \in \widehat{\mathcal{M}_{+}}$is semi-finite if $\left\{\varphi \in \mathcal{M}_{*}^{+}: m(\varphi)<+\infty\right\}$ is dense in $\mathcal{M}_{*}^{+} ;$faithful if $m(\varphi)>0$ for every non-zero $\varphi \in \mathcal{M}_{*}^{+}$.

Theorem 1.5. Let $\mathcal{M}$ be a von Neumann algebra. Each $m \in \widehat{\mathcal{M}}_{+}$has a unique spectral decomposition of the form

$$
m(\varphi)=\int_{0}^{\infty} \lambda d \varphi(e(\lambda))+\infty \varphi(p), \quad \varphi \in \mathcal{M}_{*}^{+},
$$

where $\left\{e(\lambda): \lambda \in \mathbb{R}_{+}\right\}$is an increasing family of projections in $\mathcal{M}$ which is $\sigma-$ strongly continuous from the right, and $p=1-\lim _{\lambda \rightarrow \infty} e(\lambda)$. Furthermore, $e(0)=0$ if and only if $m$ is faithful, and $p=0$ if and only if $m$ is semi-finite.

To simplify the notation, we write

$$
m=H+\infty p, \quad H=\int_{0}^{\infty} \lambda d e(\lambda),
$$

when $m$ has the form of (2). We keep the convention $0 \cdot(+\infty)=0$. Although we consider $H$ as an operator affiliated with $\mathcal{M}$, we use the following notation:

$$
\mathcal{D}\left(H^{1 / 2}\right)=\left\{\xi \in \mathfrak{H}: m\left(\omega_{\xi}\right)<+\infty\right\},
$$

as long as circumstances allow us to do this. 
Corollary 1.6. Any normal weight $\varphi$ on $\mathcal{M}$ has a unique extension, denoted again by $\varphi$, to $\widehat{\mathcal{M}_{+}}$such that

$$
\begin{aligned}
& \varphi(\lambda m)=\lambda \varphi(m), \quad \lambda \geq 0, \quad m \in \widehat{\mathcal{M}_{+}}, \\
& \varphi(m+n)=\varphi(m)+\varphi(n), \quad m, n \in \widehat{\mathcal{M}_{+}}, \\
& \varphi\left(\sup _{i} m_{i}\right)=\sup _{i} \varphi\left(m_{i}\right)
\end{aligned}
$$

for any increasing net $\left\{m_{i}\right\}$ in $\widehat{\mathcal{M}_{+}}$, where we say the net $\left\{m_{i}\right\}$ is increasing if $\left\{m_{i}(\omega)\right\}$ is increasing for every $\omega \in \mathcal{M}_{*}^{+}$.

So, we may think of the extended positive cone of $\mathcal{M}$ as $\mathcal{M}_{+}$, along with a myriad of "points at infinity" adjoined to it. There will be a different infinite point for each projection in $\mathcal{M}$. When we think about the extended positive cone in this way, it is clear why we adopt the notation in $\left(2^{\prime}\right)$.

Now, for each $m=H+\infty p$, we put

$$
\begin{aligned}
& m_{0}=(\mathbf{1}+H)^{-1}(\mathbf{1}-p), \\
& m_{\varepsilon}=H(\mathbf{1}+\varepsilon H)^{-1}(\mathbf{1}-p)+\frac{1}{\varepsilon} p, \quad \varepsilon>0 .
\end{aligned}
$$

We note that both $m_{0}$ and $m_{\varepsilon}$ are bounded.

Lemma 1.7. (i) For each $m, n \in \widehat{\mathcal{M}_{+}}$, we have the following equivalence:

$$
m \leq n \Leftrightarrow m_{0} \geq n_{0} \Leftrightarrow m_{\varepsilon} \leq n_{\varepsilon} \quad \varepsilon>0 .
$$

(ii) Let $\left\{m_{i}\right\}=\left\{H_{i}+\infty p_{i}\right\}$ be an increasing net in $\widehat{\mathcal{M}_{+}}$and $m=H+\infty p$. Then we have

$$
m_{i} \nearrow m \Leftrightarrow\left(m_{i}\right)_{0} \searrow m_{0} \Leftrightarrow\left(m_{i}\right)_{\varepsilon} \nearrow m_{\varepsilon}, \quad \varepsilon>0 .
$$

Proof. (i) Suppose $m \leq n$. Let $m=H+\infty p$ and $n=K+\infty q$. By assumption, we have $p \leq q$. Let $\mathfrak{K}=(\mathbf{1}-p) \mathfrak{H}$ and $\mathfrak{L}=(\mathbf{1}-q) \mathfrak{H}$, where $\mathfrak{H}$ denotes, as usual, the underlying Hilbert space of $\mathcal{M}$. For any $\xi \in \mathfrak{L}$, set $\eta=(\varepsilon \mathbf{1}+H)^{-1} \xi \in \mathcal{D}(H)$ and $\zeta=(\varepsilon \mathbf{1}+K)^{-1} \xi \in \mathcal{D}(K)$. We then have

$$
\begin{aligned}
\left((\varepsilon \mathbf{1}+K)^{-1} \xi \mid \xi\right)^{2}= & \left(\xi \mid(\varepsilon \mathbf{1}+K)^{-1} \xi\right)^{2}=((\varepsilon \mathbf{1}+H) \eta \mid \zeta)^{2} \\
= & \left((\varepsilon \mathbf{1}+H)^{1 / 2} \eta \mid(\varepsilon \mathbf{1}+H)^{1 / 2} \zeta\right)^{2} \\
& \left(\zeta \in \mathcal{D}(K) \subset \mathcal{D}\left(K^{1 / 2}\right) \subset \mathcal{D}\left(H^{1 / 2}\right)=\mathcal{D}\left((\varepsilon \mathbf{1}+H)^{1 / 2}\right)\right) \\
\leqq & \left\|(\varepsilon \mathbf{1}+H)^{1 / 2} \eta\right\|^{2}\left\|(\varepsilon \mathbf{1}+H)^{1 / 2} \zeta\right\|^{2} \\
= & ((\varepsilon \mathbf{1}+H) \eta \mid \eta)((\varepsilon \mathbf{1}+H) \zeta \mid \zeta) \\
\leqq & ((\varepsilon \mathbf{1}+H) \eta \mid \eta)((\varepsilon \mathbf{1}+K) \zeta \mid \zeta) \\
= & \left(\xi \mid(\varepsilon \mathbf{1}+H)^{-1} \xi\right)\left(\xi \mid(\varepsilon \mathbf{1}+K)^{-1} \xi\right)
\end{aligned}
$$

so that

$$
\left.\left((\varepsilon \mathbf{1}+K)^{-1} \xi\right) \mid \xi\right) \leqq\left((\varepsilon \mathbf{1}+H)^{-1} \xi \mid \xi\right), \quad \xi \in \mathfrak{L} .
$$

Hence we get $n_{0}\left(\omega_{\xi}\right) \leq m_{0}\left(\omega_{\xi}\right)$ for any $\xi \in \mathfrak{L}$ by setting $\varepsilon=1$. If $\xi \in \mathfrak{L}^{\perp}$, then $n_{0}\left(\omega_{\xi}\right)=0$. Thus we conclude that $n_{0} \leq m_{0}$. 
Suppose $n_{0} \leq m_{0}$. It follows that $p \leq q$. Let $\mathfrak{K}$ and $\mathfrak{L}$ be as before. The assumption means that

$$
\left((\mathbf{1}+H)^{-1} \xi \mid \xi\right) \geq\left((\mathbf{1}+K)^{-1} \xi \mid \xi\right), \quad \xi \in \mathfrak{H} .
$$

Setting $(\mathbf{1}+K)^{-1} \xi=0$ for $\xi \in \mathfrak{L}^{\perp} \cap \mathfrak{K}$, we view $(\mathbf{1}+K)^{-1}$ as an operator on $\mathfrak{K}$ and have $(\mathbf{1}+H)^{-1} \geq(\mathbf{1}+K)^{-1}$. Then we have $(\mathbf{1}+H)^{-1 / 2} \geqq(\mathbf{1}+K)^{-1 / 2}$, and

$$
\mathcal{D}\left(H^{1 / 2}\right)=(\mathbf{1}+H)^{-1 / 2} \mathfrak{K} \supset(\mathbf{1}+K)^{1 / 2} \mathfrak{K}=\mathcal{D}\left(K^{1 / 2}\right) .
$$

The argument in the first paragraph shows that

$$
\begin{aligned}
& (\mathbf{1}+H)\left(\mathbf{1}+\varepsilon(\mathbf{1}+H)^{-1}\right)^{-1}=\left(\varepsilon \mathbf{1}+(\mathbf{1}+H)^{-1}\right)^{-1} \\
& \leq\left(\varepsilon \mathbf{1}+(\mathbf{1}+K)^{-1}\right)^{-1}=(\mathbf{1}+K)\left(\mathbf{1}+\varepsilon(\mathbf{1}+K)^{-1}\right) .
\end{aligned}
$$

If $\xi \in \mathcal{D}\left(K^{1 / 2}\right)$, we have

$$
\begin{aligned}
\left\|(\mathbf{1}+K)^{1 / 2} \xi\right\|^{2} & =\lim _{\varepsilon \rightarrow 0}\left\|(\mathbf{1}+K)^{1 / 2}\left(\mathbf{1}+\varepsilon(\mathbf{1}+K)^{-1}\right)^{-1 / 2} \xi\right\|^{2} \\
& \geq \lim _{\varepsilon \rightarrow 0}\left\|(\mathbf{1}+H)^{1 / 2}\left(\mathbf{1}+\varepsilon(\mathbf{1}+H)^{-1}\right)^{-1 / 2} \xi\right\|^{2} \\
& =\left\|(\mathbf{1}+H)^{1 / 2} \xi\right\|^{2},
\end{aligned}
$$

so we conclude that $\mathbf{1}+n \geq \mathbf{1}+m$; equivalently $n \geq m$.

Now we have seen the equivalence: $m \leq n \Leftrightarrow m_{0} \geq n_{0}$. For a fixed $\varepsilon>0$, we have then

$$
\begin{aligned}
m \leq n & \Leftrightarrow \varepsilon m \leq \varepsilon n \Leftrightarrow(\varepsilon m)_{0} \geq(\varepsilon n)_{0} \\
& \Leftrightarrow \mathbf{1}-(\varepsilon m)_{0} \leq \mathbf{1}-(\varepsilon n)_{0} \\
& \Leftrightarrow m_{\varepsilon}=\frac{1}{\varepsilon}\left(\mathbf{1}-(\varepsilon m)_{0}\right) \leq \frac{1}{\varepsilon}\left(\mathbf{1}-(\varepsilon n)_{0}\right)=n_{\varepsilon} .
\end{aligned}
$$

(ii) By (i), the net $\left\{\left(m_{i}\right)_{0}\right\}$ is decreasing. If $\ell=\inf _{i}\left(m_{i}\right)_{0}$, then there exists $n \in \widehat{\mathcal{M}_{+}}$such that $n_{0}=\ell$, because $\left(m_{i}\right)_{0} \leq 1$ implies $\ell \leq \mathbf{1}$. If $m=\sup _{i} m_{i}$, then we have $m_{0} \leq\left(m_{i}\right)_{0}$, so $m_{0} \leq n_{0}$, which implies $n \leq m$ by (i). Hence $m_{0}=$ $\inf _{i}\left(m_{i}\right)_{0}=\lim \left(m_{i}\right)_{0}$. Thus we prove the equivalence: $m_{i} \nearrow m \Leftrightarrow\left(m_{i}\right)_{0} \searrow m_{0}$. Finally, the equality

$$
m_{\varepsilon}=\frac{1}{\varepsilon}\left(\mathbf{1}-(\varepsilon m)_{0}\right)
$$

gives the remaining equivalence.

Proposition 1.8. Let $\varphi$ be a faithful, semi-finite normal weight on $\mathcal{M}$, and set $\mathcal{N}=\mathcal{M}_{\varphi}$. (Here $\mathcal{M}_{\varphi}$ denotes, as is customary, the centralizer of $\varphi$ in $\mathcal{M}$.) For each $m \in \widehat{\mathcal{N}_{+}}$, set

$$
\varphi_{m}(x)=\lim _{\varepsilon \rightarrow 0} \varphi_{m_{\varepsilon}}(x), \quad x \in \mathcal{M}_{+} .
$$

(Recall that, as $m_{\varepsilon} \in \mathcal{N}_{+}=\left(\mathcal{M}_{\varphi}\right)_{+}, \varphi_{m_{\varepsilon}}:=\varphi\left(m_{\varepsilon} \cdot\right)$ gives a weight on $\mathcal{M}$.)

Then the map $m \in \widehat{\mathcal{N}}_{+} \mapsto \varphi_{m}$ is an order preserving bijection from $\widehat{\mathcal{N}}_{+}$onto the set of all $\sigma_{t}^{\varphi}$-invariant, not necessarily faithful nor semi-finite, normal weights on $\mathcal{M}$. Furthermore, we have

$$
m_{i} \nearrow m \text { in } \widehat{\mathcal{N}_{+}} \quad \Leftrightarrow \quad \varphi_{m_{i}} \nearrow \varphi_{m} \text { pointwise on } \mathcal{M}_{+} .
$$


Proof. For a fixed $x \in \mathcal{M}_{+}$, we define a normal weight $\varphi^{x}$ on $\mathcal{N}$ by

$$
\varphi^{x}(a)=\varphi\left(a^{1 / 2} x a^{1 / 2}\right), \quad a \in \mathcal{N}_{+} .
$$

If we prove the additivity of $\varphi^{x}$, then the normality follows from that of $\varphi$. Let $a, b \in \mathcal{N}_{+}$and $c=a+b$. Choose $s, t \in \mathcal{N}$ as usual so that $a^{1 / 2}=s c^{1 / 2}, b^{1 / 2}=t c^{1 / 2}$ and $s^{*} s+t^{*} t$ is the range projection of $c$. If $\varphi^{x}(c)<+\infty$, then $y=c^{1 / 2} x c^{1 / 2} \in \mathfrak{m}_{\varphi}$. (Here, $\mathfrak{m}_{\varphi}$ denotes the domain of $\varphi$, having been extended by linearity.) Hence, sys* and tyt* both belong to $\mathfrak{m}_{\varphi}$, and we get

$$
\begin{gathered}
\varphi\left(s y s^{*}\right)+\varphi\left(t y t^{*}\right)=\varphi\left(y s^{*} s\right)+\varphi\left(y t^{*} t\right)=\varphi\left(y\left(s^{*} s+t^{*} t\right)\right)=\varphi(y)=\varphi^{x}(c), \\
\varphi\left(s y s^{*}\right)=\varphi\left(a^{1 / 2} x a^{1 / 2}\right)=\varphi^{x}(a) \\
\varphi\left(t y t^{*}\right)=\varphi\left(b^{1 / 2} x b^{1 / 2}\right)=\varphi^{x}(b) .
\end{gathered}
$$

Thus, $\varphi^{x}(a)+\varphi^{x}(b)=\varphi^{x}(c)$. Now, we have

$$
s=\lim _{\varepsilon \rightarrow 0} a^{1 / 2}(c+\varepsilon \mathbf{1})^{-1 / 2}, \quad t=\lim _{\varepsilon \rightarrow 0} b^{1 / 2}(c+\varepsilon \mathbf{1})^{-1 / 2},
$$

so that

$$
\begin{aligned}
c^{1 / 2} x c^{1 / 2} & =\lim _{\varepsilon \rightarrow 0}(c+\varepsilon \mathbf{1})^{-1 / 2} c x c(c+\varepsilon \mathbf{1})^{-1 / 2} \\
& =\lim _{\varepsilon \rightarrow 0}(c+\varepsilon \mathbf{1})^{-1 / 2}(a+b) x(a+b)(c+\varepsilon \mathbf{1})^{-1 / 2} \\
& \leq 2 \lim _{\varepsilon \rightarrow 0}(c+\varepsilon \mathbf{1})^{-1 / 2}[a x a+b x b](c+\varepsilon \mathbf{1})^{-1 / 2} \\
& =2\left(s^{*} a^{1 / 2} x a^{1 / 2} s+t^{*} b^{1 / 2} x b^{1 / 2} t\right) .
\end{aligned}
$$

Therefore, if $\varphi^{x}(a)<+\infty$ and $\varphi^{x}(b)<+\infty$, then $\varphi^{x}(c)<+\infty$; hence $\varphi^{x}(a+b)=$ $\varphi^{x}(a)+\varphi^{x}(b)$ for $a, b \in \mathcal{N}_{+}$.

Now, for each $m \in \widehat{\mathcal{N}_{+}}$, we set

$$
\varphi_{m}(x)=\varphi^{x}(m), \quad x \in \mathcal{M}_{+} .
$$

This makes sense by Corollary 1.6: we have $\varphi^{x}\left(\sup _{i} m_{i}\right)=\sup _{i} \varphi^{x}\left(m_{i}\right)$ whenever $m_{i} \nearrow m$. Hence we have

$$
\varphi_{m}(x)=\varphi^{x}(m)=\lim _{\varepsilon \rightarrow 0} \varphi^{x}\left(m_{\varepsilon}\right)=\lim _{\varepsilon \rightarrow 0} \varphi_{m_{\varepsilon}}(x) .
$$

If $x_{i} \nearrow x$ in $\mathcal{M}_{+}$, then

$$
\begin{aligned}
\varphi^{x}(m) & =\sup _{\varepsilon>0} \varphi_{m_{\varepsilon}}(x)=\sup _{\varepsilon>0} \sup _{i} \varphi_{m_{\varepsilon}}\left(x_{i}\right) \\
& =\sup _{i} \sup _{\varepsilon>0} \varphi_{m_{\varepsilon}}\left(x_{i}\right)=\sup _{i} \varphi_{m}\left(x_{i}\right),
\end{aligned}
$$

so that $\varphi_{m}$ is normal. The additivity of $\varphi_{m}$ follows from the convergence in (3).

The invariance of $\varphi_{m}$ under $\left\{\sigma_{t}^{\varphi}: t \in \mathbb{R}\right\}$ follows from that of $\varphi_{m_{\varepsilon}}$. The remainder of the proof is straightforward.

We now define operator valued weights. These will generalize ordinary weights, as they are allowed to assume infinite values; also, they extend the concept of conditional expectation ("projection of norm one") in that they map from a larger von Neumann algebra onto (the extended positive cone of) a subalgebra. 
Definition 1.9. Let $\mathcal{M}$ be a von Neumann algebra and $\mathcal{N}$ a von Neumann subalgebra of $\mathcal{M}$. An operator valued weight from $\mathcal{M}$ to $\mathcal{N}$ is a map $T: \mathcal{M}_{+} \rightarrow \widehat{\mathcal{N}_{+}}$ which satisfies the following conditions:

$$
\begin{aligned}
& \text { (a) } T(\lambda x)=\lambda T(x), \quad \lambda \geq 0, \quad x \in \mathcal{M}_{+}, \\
& \text {(b) } T(x+y)=T(x)+T(y), \quad x, y \in \mathcal{M}_{+}, \\
& \text {(c) } T\left(a^{*} x a\right)=a^{*} T(x) a, \quad x \in \mathcal{M}_{+}, \quad a \in \mathcal{N} .
\end{aligned}
$$

In addition, we say that $T$ is normal if

$$
\text { (d) } T\left(x_{i}\right) \nearrow T(x) \quad \text { whenever } x_{i} \nearrow x, \quad x_{i}, x \in \mathcal{M}_{+} .
$$

As in the case of ordinary weights, we set

$$
\begin{aligned}
& \mathfrak{n}_{T}=\left\{x \in \mathcal{M}:\left\|T\left(x^{*} x\right)\right\|<+\infty\right\}, \\
& \mathfrak{m}_{T}=\mathfrak{n}_{T}^{*} \mathfrak{n}_{T}=\left\{\sum_{i=1}^{n} y_{i}^{*} x_{i}: x_{1}, \ldots, x_{n}, y_{1}, \ldots, y_{n} \in \mathfrak{n}_{T}\right\} .
\end{aligned}
$$

We state the following lemma, whose proof is standard.

Lemma 1.10. (i) $\mathfrak{m}_{T}$ is spanned by its positive part, viz.,

$$
\mathfrak{m}_{T}^{+}=\left\{x \in \mathcal{M}_{+}:\|T(x)\|<+\infty\right\} .
$$

(ii) $\mathfrak{m}_{T}$ and $\mathfrak{n}_{T}$ are two sided modules over $\mathcal{N}$.

(iii) $T$ has a unique linear extension $\dot{T}: \mathfrak{m}_{T} \rightarrow \mathcal{N}$, which enjoys the module map property

$$
\dot{T}(a x b)=a \dot{T}(x) b, \quad a, b \in \mathcal{N}, \quad x \in \mathfrak{m}_{T} .
$$

In particular, if $T(\mathbf{1})=\mathbf{1}$, then $T$ is a projection of norm one (conditional expectation) from $\mathcal{M}$ onto $\mathcal{N}$.

In the sequel, we shall not distinguish $T$ and $\dot{T}$ unless we need to.

Definition 1.11. We say that $T$ is semi-finite if $\mathfrak{n}_{T}$ is $\sigma$-weakly dense in $\mathcal{M}$; faithful if $T\left(x^{*} x\right) \neq 0$ for $x \neq 0$. We denote by $\mathcal{W}(\mathcal{M}, \mathcal{N})\left(\operatorname{resp} . \mathcal{W}_{0}(\mathcal{M}, \mathcal{N})\right)$ the set of (resp. faithful, semi-finite) normal operator valued weights from $\mathcal{M}$ to $\mathcal{N}$. In the case that $\mathcal{N}=\mathbb{C}$, we write $\mathcal{W}(\mathcal{M})\left(\right.$ resp. $\left.\mathcal{W}_{0}(\mathcal{M})\right)$ for $\left(\mathcal{W}(\mathcal{M}, \mathbb{C})\left(\operatorname{resp} . \mathcal{W}_{0}(\mathcal{M}, \mathbb{C})\right)\right.$.

Note that if $T: \mathcal{M}_{+} \rightarrow \widehat{\mathcal{N}_{+}}$is a normal operator valued weight, it can be extended to a normal "linear" map from $\widehat{\mathcal{M}}_{+} \rightarrow \widehat{\mathcal{N}}_{+}$. Therefore, if $\mathcal{P} \subset \mathcal{N} \subset \mathcal{M}$ is an inclusion of von Neumann algebras, and if $T \in \mathcal{W}(\mathcal{M}, \mathcal{N})$ and $S \in \mathcal{W}(\mathcal{N}, \mathcal{P})$, then we have $S \circ T \in \mathcal{W}(\mathcal{M}, \mathcal{P})$.

Proposition 1.12. If $\mathcal{P} \subset \mathcal{N} \subset \mathcal{M}$ are von Neumann subalgebras and if $T \in$ $\mathcal{W}_{0}(\mathcal{M}, \mathcal{N})$ and $S \in \mathcal{W}_{0}(\mathcal{N}, \mathcal{P})$, then $S \circ T \in \mathcal{W}_{0}(\mathcal{M}, \mathcal{P})$.

Proof. The only non-trivial part is the semi-finiteness of $S \circ T$. If $x \in \mathfrak{n}_{T}$, then $T\left(x^{*} x\right) \in \mathcal{N}_{+}$. Choose a net $\left\{a_{i}\right\}$ in $\mathfrak{n}_{S}$ such that $a_{i} \rightarrow \mathbf{1} \sigma$-strongly. Then we have

$$
S \circ T\left(a_{i}^{*} x^{*} x a_{i}\right)=S\left(a_{i}^{*} T\left(x^{*} x\right) a_{i}\right) \leq\left\|T\left(x^{*} x\right)\right\| S\left(a_{i}^{*} a_{i}\right),
$$

so that $x a_{i} \in \mathfrak{n}_{S \circ T}$. Hence $\mathfrak{n}_{S \circ T}$ is $\sigma$-strongly dense in $\mathcal{M}$ because $\mathfrak{n}_{T}$ is.

Proposition 1.13. Suppose $\mathcal{N}, \mathcal{M}$ are von Neumann algebras, $\mathcal{N} \subset \mathcal{M}$ and $T \in$ $\mathcal{W}_{0}(\mathcal{M}, \mathcal{N})$.

(i) $\dot{T}\left(\mathfrak{m}_{T}\right)$ is a $\sigma$-weakly dense 2 -sided ideal of $\mathcal{N}$. 
(ii) After extending $T$ to $\dot{T}: \widehat{\mathcal{M}_{+}} \rightarrow \widehat{\mathcal{N}_{+}}$, we have

$$
T\left(\widehat{\mathcal{M}_{+}}\right)=\widehat{\mathcal{N}_{+}} \text {. }
$$

Proof. (i) From the module map property of $T$,

$$
\dot{T}(a x b)=a \dot{T}(x) b, \quad a, b \in \mathcal{N}, x \in \mathfrak{m}_{T},
$$

it follows that $\dot{T}\left(\mathfrak{m}_{T}\right)$ is an ideal of $\mathcal{N}$. Let $z$ denote the greatest projection of the $\sigma$-weak closure of $\dot{T}\left(\mathfrak{m}_{T}\right)$, which is central in $\mathcal{N}$. Assume $z \neq \mathbf{1}$. As $\mathfrak{n}_{T}$ is $\sigma$-weakly dense in $\mathcal{M}$, there exists $x \in \mathfrak{n}_{T}$ with $x(\mathbf{1}-z) \neq 0$, so that $(\mathbf{1}-z) x^{*} x(\mathbf{1}-z) \in$ $\mathfrak{m}_{T} \backslash\{0\}$, and as $T$ is faithful

$$
0 \neq T\left((\mathbf{1}-z) x^{*} x(\mathbf{1}-z)\right)=(\mathbf{1}-z) T\left(x^{*} x\right)(\mathbf{1}-z)=0,
$$

which is a contradiction. Hence $z=\mathbf{1}$, which means that $\dot{T}\left(\mathfrak{m}_{T}\right)$ is $\sigma$-weakly dense in $\mathcal{N}$.

(ii) Take any $b \in \dot{T}\left(\mathfrak{m}_{T}\right)_{+}$. Then $b$ is of the form $b=\dot{T}(h), h \in \mathfrak{m}_{T}$. Replacing $h$ by $\frac{1}{2}\left(h+h^{*}\right), h$ can be chosen to be self-adjoint. Now, by Lemma (1.10), we know there exist $a_{1}, a_{2} \in \mathfrak{m}_{T}^{+}$such that $b=T\left(a_{1}\right)-T\left(a_{2}\right)$. Then, we have $b \leq T\left(a_{1}\right)$, so we can find $s \in \mathcal{N}$ such that $b=s T\left(a_{1}\right) s^{*}$. With $a=s a_{1} s^{*}$, we have $b=T(a)$, $a \in \mathfrak{m}_{T}^{+}$. Hence $\dot{T}\left(\mathfrak{m}_{T}\right)_{+}=\dot{T}\left(\mathfrak{m}_{T}^{+}\right)$.

Now, let $\left\{b_{\lambda}\right\}_{\lambda \in \Lambda}$ be a family in the positive cone of the ideal $\dot{T}\left(\mathfrak{m}_{T}\right)_{+}$, maximal with respect to the property $\sum_{\lambda \in \Lambda} b_{\lambda} \leq \mathbf{1}$. By this maximality, and an appeal to the Kaplansky density theorem, we may conclude that $\sum_{\lambda \in \Lambda} b_{\lambda}=\mathbf{1}$ in the $\sigma$-strong topology. Every $y \in \mathcal{N}_{+}$is then of the form $y=\sum_{\lambda \in \Lambda} y^{\frac{1}{2}} b_{\lambda} y^{\frac{1}{2}}$, so that we have $y=\sum_{\lambda \in \Lambda} T\left(x_{\lambda}\right)$ with $\left\{x_{\lambda}\right\} \subset \mathfrak{m}_{T}^{+}$.

Finally, suppose $z \in \widehat{\mathcal{N}_{+}}$. From (2), it follows that there exists a sequence $\left\{y_{n}\right\} \subset \mathcal{N}_{+}$such that $y_{n} \nearrow z$. Set $z_{1}:=y_{1}$ and $z_{n}:=y_{n}-y_{n-1}, n \geq 2$. Then we have $z=\sum_{n=1}^{\infty} z_{n}$. Each $z_{n}$ can be written $z_{n}=\sum T\left(\left(x_{n}\right)_{\lambda}\right)$ with $\left\{\left(x_{n}\right)_{\lambda}\right\} \subset \mathfrak{m}_{T}^{+}$. Hence we have

$$
z=T\left(\sum_{n=1}^{\infty} \sum_{\lambda}\left(x_{n}\right)_{\lambda}\right)
$$

where $T$ has been extended to $\widehat{\mathcal{M}_{+}}$. Thus $T$ maps $\widehat{\mathcal{M}_{+}}$onto $\widehat{\mathcal{N}_{+}}$.

\section{The Main Result}

We now restate our main theorem. It is obvious how it generalizes Takesaki's theorem [Tak1] which states that a necessary and sufficient condition for the existence of a normal conditional expectation $\mathcal{E}: \mathcal{M} \rightarrow \mathcal{N}$ with respect to a normal, faithful and semi-finite weight $\varphi$ is the invariance of $\mathcal{N}$ under the modular automorphism group $\left\{\sigma_{t}^{\varphi}\right\}$.

Theorem 2.1. Let $\mathcal{N} \subset \mathcal{M}$ be von Neumann algebras. There exists a faithful semi-finite normal operator valued weight $T: \mathcal{M}_{+} \rightarrow \widehat{\mathcal{N}_{+}}$if and only if there exist faithful semi-finite normal weights $\tilde{\varphi}$ on $\mathcal{M}$ and $\varphi$ on $\mathcal{N}$ such that

$$
\sigma_{t}^{\varphi}(x)=\sigma_{t}^{\tilde{\varphi}}(x), \quad x \in \mathcal{N} .
$$

If this condition is satisfied, then $T$ can be chosen in such a way that $\tilde{\varphi}=\varphi \circ T$; moreover, $T$ is uniquely determined by this identity. 
In order to prove this, we begin first with a lemma which is of independent interest; note its measure-theoretic flavor. A proof similar to the one included herein can also be found in [Haa1]; we have included it for completeness.

Lemma 2.2. An extended positive real valued function $m: \mathcal{M}_{*}^{+} \rightarrow[0,+\infty]$ having the properties

$$
\begin{aligned}
m(\lambda \varphi) & =\lambda m(\varphi), \quad \lambda \geq 0, \varphi \in \mathcal{M}_{*}^{+}, \\
m(\varphi+\psi) & =m(\varphi)+m(\psi), \quad \varphi, \psi \in \mathcal{M}_{*}^{+},
\end{aligned}
$$

is lower semi-continuous, and hence a member of the extended positive cone $\widehat{\mathcal{M}}_{+}$, if and only if $m$ is countably additive in the sense that

$$
m\left(\sum_{n=1}^{\infty} \varphi_{n}\right)=\sum_{n=1}^{\infty} m\left(\varphi_{n}\right)
$$

for every $\left\{\varphi_{n}\right\} \subset \mathcal{M}_{*}^{+}$with $\sum_{n=1}^{\infty}\left\|\varphi_{n}\right\|<+\infty$.

Proof. The "only if" part is trivial, so we prove the "if". Suppose $m$ is countably additive. Represent $\mathcal{M}$ on a Hilbert space $\mathfrak{H}$ and consider $\bar{m}: \omega \in \mathcal{L}(\mathfrak{H})_{*}^{+} \mapsto$ $m\left(\left.\omega\right|_{\mathcal{M}}\right) \in[0,+\infty]$. We claim first that $m$ is lower semi-continuous if $\bar{m}$ is. Suppose $\bar{m}$ is lower semi-continuous. Then there exists a unique pair $\{A, \mathfrak{K}\}$ of a closed subspace $\mathfrak{K}$ of $\mathfrak{H}$ and a positive self-adjoint operator $A$ on $\mathfrak{K}$ such that (1) holds. As $\left.u \omega u^{*}\right|_{\mathcal{M}}=\left.\omega\right|_{\mathcal{M}}$ for every $u \in \mathcal{U}\left(\mathcal{M}^{\prime}\right)$, it follows that $u^{*} \bar{m} u=\bar{m}$, so that $\{A, \mathfrak{K}\}$ is affiliated to $\mathcal{M}$, and $m=m_{A}$. Thus, $m$ is a member of $\widehat{\mathcal{M}_{+}}$. Therefore, it suffices to prove the lemma for $\mathcal{M}=\mathcal{L}(\mathfrak{H})$. Replacing $m$ by $m^{\prime}$ defined by $m^{\prime}(\varphi)=m(\varphi)+\varphi(\mathbf{1})$, we may assume $m(\varphi) \geq\|\varphi\|, \varphi \in \mathcal{M}_{*}^{+}$. As $\mathcal{M}_{*}=L^{1}(\mathcal{M}, \operatorname{Tr})$ is an ideal of $\mathcal{M}$, we can define a map $\varphi: x \in \mathcal{M}_{+} \mapsto[0,+\infty]$ by

$$
\varphi(x)= \begin{cases}m\left(\omega_{x}\right), & x \in L^{1}(\mathcal{M}, \operatorname{Tr})_{+}, \\ +\infty, & \text { otherwise }\end{cases}
$$

where $\omega_{x}(a)=\operatorname{Tr}(a x), a \in \mathcal{M}, x \in L^{1}(\mathcal{M}, \operatorname{Tr})$. Then $\varphi$ is a weight on $\mathcal{M}$, and $\varphi \geq \operatorname{Tr}$. Let $\left\{x_{i}\right\}_{i \in I}$ be a family of positive operators with $x=\sum x_{i} \in \mathcal{M}_{+}$. If $\operatorname{Tr}(x)=+\infty$, then $\sum_{i} \operatorname{Tr}\left(x_{i}\right)=\infty$ and

$$
\infty=\sum \operatorname{Tr}\left(x_{i}\right) \leq \sum \varphi\left(x_{i}\right) \leq \varphi(x),
$$

so that $\varphi(x)=\infty=\sum \varphi\left(x_{i}\right)$. If $\operatorname{Tr}(x)<+\infty$, then $\sum \operatorname{Tr}\left(x_{i}\right)<+\infty$, so that $x_{i} \neq 0$ for at most countably infinitely many $i$ 's. Hence we have

$$
\begin{aligned}
\sum_{i \in I} \varphi\left(x_{i}\right) & =\sum_{i} m\left(\omega_{x_{i}}\right)=m\left(\sum \omega_{x_{i}}\right) \\
& =m\left(\omega_{x}\right)=\varphi(x) .
\end{aligned}
$$

Thus, $\varphi$ is a completely additive weight on $\mathcal{M}$, and thus $\varphi$ is normal. If $\left\{x_{n}\right\}$ is a sequence in $L^{1}(\mathcal{M}, \operatorname{Tr})_{+}$with $\lim _{n \rightarrow \infty}\left\|x-x_{n}\right\|_{1}=0$, then $\left\{x_{n}\right\}$ converges to $x$ $\sigma$-weakly, so that

$$
m\left(\omega_{x}\right)=\varphi(x) \leq \liminf _{n \rightarrow \infty} \varphi\left(x_{n}\right)=\liminf _{n \rightarrow \infty} m\left(\omega_{x_{n}}\right) .
$$

Hence $m$ is lower semi-continuous. 
We will also need the following lemma, which appears without proof. It can be demonstrated by an application of one of the results in the theory of the cocycle derivative. (See, for example, [Tak3].)

Lemma 2.3. Let $\varphi \in \mathcal{W}(\mathcal{M})$, and let $\left\{a_{n}\right\}$ be a sequence in $\mathcal{M}_{s(\varphi)}$. (Here, as usual, $\mathcal{M}_{s(\varphi)}$ represents the reduced algebra.) Then the following two conditions are equivalent:

(i) $\varphi(x)=\sum_{n=1}^{\infty} \varphi\left(a_{n} x a_{n}^{*}\right), \quad x \in \mathcal{M}_{+}$.

(ii) $\left\{a_{n}\right\} \subset \mathcal{D}\left(\sigma_{-i / 2}^{\varphi}\right)$ and $\sum_{n=1}^{\infty} \sigma_{-i / 2}^{\varphi}\left(a_{n}\right)^{*} \sigma_{-i / 2}^{\varphi}\left(a_{n}\right)=s(\varphi)$.

We can now proceed with the proof of our main theorem.

Proof of Theorem 2.1. Suppose that $\mathcal{N} \subset \mathcal{M}$ are von Neumann algebras, and

$$
\sigma_{t}^{\tilde{\varphi}}(x)=\sigma_{t}^{\varphi}(x), \quad x \in \mathcal{N},
$$

for some $\tilde{\varphi} \in \mathcal{W}_{0}(\mathcal{M})$ and $\varphi \in \mathcal{W}_{0}(\mathcal{N})$. We fix $\tilde{\varphi}$ and $\varphi$. For each $\psi \in \mathcal{W}(\mathcal{N})$, we have the cocycle derivative $u_{t}=(D \psi: D \varphi)_{t} \in \mathcal{N}, t \in \mathbb{R}$. By a result of Masuda [Mas], there corresponds $\tilde{\psi} \in \mathcal{W}(\mathcal{M})$ such that $s(\tilde{\psi})=s(\psi)$ and $(D \tilde{\psi}: D \tilde{\varphi})_{t}=$ $u_{t}=(D \psi: D \varphi)_{t}$. As we have

$$
\begin{aligned}
(D(\lambda \psi): D \varphi)_{t} & =\lambda^{i t}(D \psi: D \varphi)_{t}=\lambda^{i t}(D \tilde{\psi}: D \tilde{\varphi})_{t} \\
& =(D(\lambda \tilde{\psi}): D \tilde{\varphi})_{t},
\end{aligned}
$$

we obtain $\widetilde{\lambda \psi}=\lambda \tilde{\psi}, \lambda>0$.

By the chain rule for cocycle derivatives, we have $\left(D \tilde{\psi}_{1}: D \tilde{\psi}_{2}\right)_{t}=\left(D \psi_{1}: D \psi_{2}\right)_{t}$ for any $\psi_{1} \in \mathcal{W}(\mathcal{N})$ and $\psi_{2} \in \mathcal{W}_{0}(\mathcal{N})$. Let $\left\{\psi_{n}\right\}$ be a sequence in $\mathcal{N}_{*}^{+}$satisfying $\psi=\sum_{n=1}^{\infty} \psi_{n} \in \mathcal{N}_{*}^{+}$. Let $u_{t}^{n}=\left(D \psi_{n}: D \psi\right)_{t}$ (when we restrict our consideration to $\mathcal{N}_{s(\psi)}$ and $\mathcal{M}_{s(\tilde{\psi})}$ ). Then we have seen that $u_{-i / 2}^{n}$ is defined, and

$$
\psi_{n}(x)=\psi\left(\left(u_{-i / 2}^{n}\right)^{*} x\left(u_{-i / 2}^{n}\right)\right), \quad x \in \mathcal{N} .
$$

Therefore, the previous lemma implies

$$
\begin{aligned}
s(\psi) & =\sum_{n=1}^{\infty} \sigma_{-i / 2}^{\psi}\left(\left(u_{-i / 2}^{n}\right)^{*}\right)^{*} \sigma_{-i / 2}^{\psi}\left(\left(u_{-i / 2}^{n}\right)^{*}\right) \\
& =\sum_{n=1}^{\infty} \sigma_{i / 2}^{\psi}\left(u_{-i / 2}^{n}\right) \sigma_{i / 2}^{\psi}\left(u_{-i / 2}^{n}\right)^{*} \\
& =\sum_{n=1}^{\infty} \sigma_{i / 2}^{\tilde{\psi}}\left(\left(D \tilde{\psi}_{n}: D \tilde{\psi}\right)_{-i / 2}\right) \sigma_{i / 2}^{\tilde{\psi}}\left(\left(D \tilde{\psi}_{n}: D \tilde{\psi}\right)_{-i / 2}\right)^{*} .
\end{aligned}
$$

As $s(\psi)=s(\tilde{\psi})$, the above calculation shows that

$$
\tilde{\psi}(x)=\sum_{n=1}^{\infty} \tilde{\psi}_{n}(x), \quad x \in \mathcal{M}_{+} .
$$

This shows that the map $\psi \in \mathcal{N}_{*}^{+} \mapsto \tilde{\psi} \in \mathcal{W}(\mathcal{M})$ is homogeneous and countably additive. Hence, the map $\psi \in \mathcal{N}_{*}^{+} \mapsto \tilde{\psi}(x) \in[0,+\infty], x \in \mathcal{M}_{+}$, gives rise to a map $T: \mathcal{M}_{+} \rightarrow \widehat{\mathcal{N}_{+}}$. 
Note that we also have the following: for every $u \in \mathcal{U}(\mathcal{N})$,

$$
\begin{aligned}
\left(D\left(u \psi u^{*}\right)^{\sim}: D \tilde{\varphi}\right)_{t} & =\left(D\left(u \psi u^{*}\right): D \varphi\right)_{t} \\
& =u(D \psi: D \varphi)_{t} \sigma_{t}^{\varphi}\left(u^{*}\right)=u(D \tilde{\psi}: D \tilde{\varphi})_{t} \sigma_{t}^{\tilde{\varphi}}\left(u^{*}\right),
\end{aligned}
$$

so that $\left(u \psi u^{*}\right)^{\sim}=u \tilde{\psi} u^{*}, u \in \mathcal{U}(\mathcal{N})$.

We now want to show that

$$
T\left(a x a^{*}\right)=a T(x) a^{*}, \quad a \in \mathcal{N}, x \in \mathcal{M}_{+} .
$$

First, we observe that if $a \in \mathcal{N}_{\psi}$ with $\psi$ faithful, then $\left(D a^{*} \psi a: D \psi\right)_{t}=\left(a^{*} a\right)^{i t}$. Here $\left(a^{*} a\right)^{i t}$ should be considered in the reduced algebra $\mathcal{N}_{s_{r}(a)}$, with $s_{r}(a)$ denoting the right support of $a$, so that $\left(\widetilde{a^{*} \psi a}: D \tilde{\psi}\right)_{t}=\left(D a^{*} \psi a: D \psi\right)_{t}=\left(a^{*} a\right)^{i t}$; hence $\widetilde{a^{*} \psi a}=a^{*} \tilde{\psi} a$. If $\psi$ is not faithful, then we consider $\psi^{\prime \prime}=\psi+\psi^{\prime}$ with $\psi^{\prime} \in \mathcal{W}(\mathcal{N})$ such that $s\left(\psi^{\prime}\right)=\mathbf{1}-s(\psi)$; we apply the above argument to $\psi^{\prime \prime}$, and conclude that

$$
\widetilde{a^{*} \psi a}=a^{*} \tilde{\psi} a, \quad a \in \mathcal{N}_{\psi} .
$$

Now, we repeat the preceding argument using $\mathcal{N} \otimes M_{2}(\mathbb{C})$ and $\mathcal{M} \otimes M_{2}(\mathbb{C})$ (with $\psi \otimes \operatorname{Tr}$ and $\tilde{\psi} \otimes \operatorname{Tr}$ ), and observe that $\sigma_{t}^{\psi \otimes \operatorname{Tr}}(x)=\sigma_{t}^{\tilde{\psi} \otimes \operatorname{Tr}}(x)$ for every $x \in \mathcal{N} \otimes M_{2}(\mathbb{C})$. This implies that there exists a map $S:\left(\mathcal{M} \otimes M_{2}\right)_{+} \rightarrow\left(\widehat{\mathcal{\otimes} M_{2}}\right)_{+}$such that

$$
(\psi \otimes \operatorname{Tr}) \circ S(x)=(\widetilde{\psi \otimes \operatorname{Tr}})(x), \quad x \in\left(\mathcal{M} \otimes M_{2}\right)_{+} .
$$

As $\mathbb{C} \otimes M_{2} \subset \mathcal{N}_{\psi \otimes \operatorname{Tr}}$ for any faithful $\psi$, we have $\widetilde{\psi \otimes \operatorname{Tr}}=\tilde{\psi} \otimes \operatorname{Tr}$. If $a \in \mathcal{N}$ is such that $\|a\| \leq 1$, we set

$$
u=\left[\begin{array}{cc}
a & \left(\mathbf{1}-a a^{*}\right)^{\frac{1}{2}} \\
-\left(\mathbf{1}-a^{*} a\right)^{\frac{1}{2}} & a^{*}
\end{array}\right] \in \mathcal{N} \otimes M_{2}
$$

then $u$ is unitary. Therefore, we have

$$
u^{*}(\widetilde{\psi \otimes \operatorname{Tr}}) u=u^{*}(\tilde{\psi} \otimes \operatorname{Tr}) u .
$$

With $\left\{e_{i j}\right\}$ the standard matrix unit of $M_{2}$, we have

$$
\left[\left(\mathbf{1} \otimes e_{i j}\right)^{*}(\psi \otimes \operatorname{Tr})\left(\mathbf{1} \otimes e_{i j}\right)\right]^{\sim}=\left(\mathbf{1} \otimes e_{i j}\right)^{*}(\tilde{\psi} \otimes \operatorname{Tr})\left(\mathbf{1} \otimes e_{i j}\right)
$$

since $\left(\mathbf{1} \otimes e_{i j}\right) \in\left(\mathcal{N} \otimes M_{2}\right)_{\psi \otimes \operatorname{Tr}}$. Hence we conclude that

$$
\begin{array}{r}
{\left[\left(\mathbf{1} \otimes e_{11}\right) u^{*}\left(\mathbf{1} \otimes e_{11}\right)(\psi \otimes \operatorname{Tr})\left(\mathbf{1} \otimes e_{11}\right) u\left(\mathbf{1} \otimes e_{11}\right)\right]^{\sim}} \\
=\left(a \otimes e_{11}\right)^{*}(\tilde{\psi} \otimes \operatorname{Tr})\left(a \otimes e_{11}\right),
\end{array}
$$

i.e.,

$$
\left[\left(a^{*} \otimes e_{11}\right)(\psi \otimes \operatorname{Tr})\left(a \otimes e_{11}\right)\right]^{\sim}=\left(a^{*} \otimes e_{11}\right)(\tilde{\psi} \otimes \operatorname{Tr})\left(a \otimes e_{11}\right) .
$$

This means that

$$
\widetilde{a^{*} \psi a}=a^{*} \tilde{\psi} a
$$

for every $a \in \mathcal{N}$ with $\|a\| \leq 1$, which gives us (5). Therefore $T$ is an operator valued weight of $\mathcal{M}_{+}$onto $\widehat{\mathcal{N}_{+}}$such that $\tilde{\psi}=\psi \circ T, \psi \in \mathcal{W}_{0}(\mathcal{N})$. As $\tilde{\psi}$ is faithful for any $\psi \in \mathcal{W}_{0}(\mathcal{N}), T$ is faithful.

The semi-finiteness and uniqueness of $T$, together with the converse, follow from the next lemma. 
Lemma 2.4. Let $\mathcal{N} \subset \mathcal{M}$ be von Neumann algebras, and let $T: \mathcal{M}_{+} \rightarrow \widehat{\mathcal{N}_{+}}$be a normal operator valued weight.

(i) If $\psi \circ T$ is semi-finite for some $\psi \in \mathcal{W}_{0}(\mathcal{N})$, then $T$ is semi-finite.

(ii) If $\psi \in \mathcal{W}_{0}(\mathcal{N})$ and $S \in \mathcal{W}(\mathcal{M}, \mathcal{N})$ satisfies $\psi \circ T=\psi \circ S$, then $T=S$.

(iii) If $T \in \mathcal{W}_{0}(\mathcal{M}, \mathcal{N})$, then

$$
\sigma_{t}^{\psi \circ T}(x)=\sigma_{t}^{\psi}(x), \quad x \in \mathcal{N} .
$$

Proof. (i) Set $\tilde{\psi}=\psi \circ T$. Let $h \in \mathfrak{m}_{\tilde{\psi}}^{+}$, and let

$$
T(h)=\int_{0}^{\infty} \lambda d e(\lambda)+\infty p
$$

be its spectral decomposition. As $\psi \circ T(h)<+\infty$, and $\psi$ is faithful, we have $p=0$. Hence $e(\lambda) \nearrow \mathbf{1}$ as $\lambda \nearrow \infty$, so that $e(\lambda) h e(\lambda) \rightarrow h$ strongly as $\lambda \nearrow \infty$, and $T(e(\lambda) h e(\lambda))=e(\lambda) T(h) e(\lambda) \in \mathcal{N}_{+}$; hence $e(\lambda) h e(\lambda) \in \mathfrak{m}_{T}^{+}$. Therefore $\mathfrak{m}_{T}^{+}$is $\sigma$-strongly dense in $\mathcal{M}_{+}$.

(ii) For any $x \in \mathcal{M}_{+}$and $a \in \mathcal{N}$, we have

$$
\begin{aligned}
\psi\left(a^{*} T(x) a\right) & =\psi\left(T\left(a^{*} x a\right)\right)=\psi\left(S\left(a^{*} x a\right)\right) \\
& =\psi\left(a^{*} S(x) a\right),
\end{aligned}
$$

so that $a \psi a^{*} \circ T=a \psi a^{*} \circ S$ for any $a \in \mathcal{N}$. Therefore, if $x \in \mathfrak{m}_{T}^{+} \cap \mathfrak{m}_{S}^{+}$, then $T(x)=S(x)$, since $\left\{a \psi a^{*}: a \in \mathfrak{n}_{\psi}\right\}$ is a dense subset of $\mathcal{N}_{*}^{+}$. For a general $x \in \mathcal{M}_{+}$, consider the spectral decompositions

$$
\begin{aligned}
& m=S(x)=\int_{0}^{\infty} \lambda d e(\lambda)+\infty p \in \widehat{\mathcal{N}_{+}}, \\
& n=T(x)=\int_{0}^{\infty} \lambda d f(\lambda)+\infty q \in \widehat{\mathcal{N}_{+} .}
\end{aligned}
$$

Then we have $e(\lambda) x e(\lambda) \in \mathfrak{m}_{S}^{+} \cap \mathfrak{m}_{T}^{+}$and

$$
\begin{aligned}
m e(\lambda) & =e(\lambda) S(x) e(\lambda)=S(e(\lambda) x e(\lambda)) \\
& =T(e(\lambda) x e(\lambda))=e(\lambda) T(x) e(\lambda) \\
& =e(\lambda) n e(\lambda) ;
\end{aligned}
$$

similarly

$$
n f(\lambda)=f(\lambda) m f(\lambda), \quad \lambda \geq 0 .
$$

Hence we have, for every $\xi \in \bigcup_{\lambda \geq 0} e(\lambda) \mathfrak{H}_{\psi}$,

$$
\left\|m^{\frac{1}{2}} \xi\right\|^{2}=m\left(\omega_{\xi}\right)=n\left(\omega_{\xi}\right)=\left\|n^{\frac{1}{2}} \xi\right\|^{2} ;
$$

similarly

$$
\left\|m^{\frac{1}{2}} \xi\right\|^{2}=\left\|n^{\frac{1}{2}} \xi\right\|^{2}, \quad \xi \in \bigcup_{\lambda \geq 0} f(\lambda) \mathfrak{H}_{\psi} .
$$

But $m^{\frac{1}{2}}$ (resp., $n^{\frac{1}{2}}$ ) is essentially self-adjoint on $\bigcup e(\lambda) \mathfrak{H}_{\psi}\left(\right.$ resp., $\left.\bigcup f(\lambda) \mathfrak{H}_{\psi}\right)$, so we get

$$
\left\|m^{\frac{1}{2}} \xi\right\|^{2}=\left\|n^{\frac{1}{2}} \xi\right\|^{2}, \quad \xi \in \mathcal{D}\left(m^{\frac{1}{2}}\right) \cap \mathcal{D}\left(n^{\frac{1}{2}}\right)
$$


Hence there exists a partial isometry $u \in \mathcal{N}$ such that $m^{\frac{1}{2}}=u n^{\frac{1}{2}}$ and $n^{\frac{1}{2}}=u^{*} m^{\frac{1}{2}}$; the uniqueness of the polar decomposition implies $m^{\frac{1}{2}}=n^{\frac{1}{2}}$ and $(\mathbf{1}-p)=(\mathbf{1}-q)$. Thus $m=n$ as elements of $\widehat{\mathcal{N}_{+}}$. Hence $S=T$.

(iii) We prove that $\mathcal{G}\left(\sigma_{-i}^{\psi}\right) \subset \mathcal{G}\left(\sigma_{-i}^{\psi \circ T}\right)$. (Here, $\mathcal{G}\left(\sigma_{-i}^{\psi}\right)$ represents the graph of the (densely-defined) map $\sigma_{-i}^{\psi}$.) Let $\tilde{\psi}=\psi \circ T$, and $(a, b) \in \mathcal{G}\left(\sigma_{-i}^{\psi}\right)$. As $a \in \mathcal{D}\left(\sigma_{-i / 2}^{\psi}\right)$ and $b^{*} \in \mathcal{D}\left(\sigma_{-i / 2}^{\psi}\right)=\mathcal{D}\left(\sigma_{i / 2}^{\psi}\right)^{*}$, there exists $M \geq 0$ such that for every $x \in \mathcal{N}_{+}$

$$
\psi\left(a x a^{*}\right) \leq M^{2} \psi(x), \quad \psi\left(b^{*} x b\right) \leq M^{2} \psi(x) .
$$

Taking increasing limits, we see that the above inequalities are valid for every $x \in \widehat{\mathcal{N}_{+}}$, so that

$$
\tilde{\psi}\left(a x a^{*}\right) \leq M^{2} \tilde{\psi}(x), \quad \tilde{\psi}\left(b^{*} x b\right) \leq M^{2} \tilde{\psi}(x)
$$

for every $x \in \mathcal{M}_{+} ;$therefore $\mathfrak{n}_{\tilde{\psi}} a^{*} \subset \mathfrak{n}_{\tilde{\psi}}, \mathfrak{n}_{\tilde{\psi}} b \subset \mathfrak{n}_{\tilde{\psi}}$ and

$$
\begin{aligned}
\left\|\eta_{\tilde{\psi}}\left(x a^{*}\right)\right\| & \leq M\left\|\eta_{\tilde{\psi}}(x)\right\|, \\
\left\|\eta_{\tilde{\psi}}(x b)\right\| & \leq M\left\|\eta_{\tilde{\psi}}(x)\right\|, \quad x \in \mathfrak{n}_{\tilde{\psi}} .
\end{aligned}
$$

We will prove that $(a, b) \in \mathcal{G}\left(\sigma_{-i}^{\tilde{\psi}}\right)$. By invoking a result from the theory of cocycle derivatives [Tak3], it suffices to show that

$$
\tilde{\psi}(a x)=\tilde{\psi}(x b), \quad x \in \mathfrak{m}_{\tilde{\psi}} .
$$

Fix $x_{0}=y_{0}^{*} z_{0}$ with $y_{0}, z_{0} \in \mathfrak{n}_{\tilde{\psi}} \cap \mathfrak{n}_{T}$. As $\mathfrak{n}_{\tilde{\psi}} a^{*} \subset \mathfrak{n}_{\tilde{\psi}}$, and because $\mathfrak{n}_{T}$ is a right $\mathcal{N}$-module, we have

$$
a x_{0}=\left(y_{0} a^{*}\right)^{*} z_{0} \in\left(\mathfrak{n}_{\tilde{\psi}} \cap \mathfrak{n}_{T}\right)^{*}\left(\mathfrak{n}_{\tilde{\psi}} \cap \mathfrak{n}_{T}\right) \subset \mathfrak{m}_{\tilde{\psi}} \cap \mathfrak{m}_{T} .
$$

Similarly, we get

$$
x_{0} b=y_{0}^{*}\left(z_{0} b\right) \in \mathfrak{m}_{\tilde{\psi}} \cap \mathfrak{m}_{T} .
$$

Since $(a, b) \in \mathcal{G}\left(\sigma_{-i}^{\psi}\right)$, we have

$$
\begin{aligned}
\psi \circ \dot{T}\left(a x_{0}\right) & =\psi\left(a \dot{T}\left(x_{0}\right)\right)=\psi\left(\dot{T}\left(x_{0}\right) b\right) \\
& \left.=\psi \circ \dot{T}\left(x_{0} b\right)\right),
\end{aligned}
$$

so that

$$
\tilde{\psi}\left(a x_{0}\right)=\tilde{\psi}\left(x_{0} b\right) .
$$

Now suppose $x=y^{*} z$ with $y, z \in \mathfrak{n}_{\tilde{\psi}}$. Since $\psi \circ T\left(y^{*} y\right)<+\infty$, we have the spectral decomposition of $T\left(y^{*} y\right)$

$$
T\left(y^{*} y\right)=\int_{0}^{\infty} \lambda d e(\lambda) .
$$

For any $\lambda>0$, we have $y e(\lambda) \in \mathfrak{n}_{T}$ and

$$
\begin{gathered}
\psi \circ T\left(e(\lambda) y^{*} y e(\lambda)\right)=\psi\left(e(\lambda) T\left(y^{*} y\right) e(\lambda)\right) \\
\leq \psi \circ T\left(y^{*} y\right)<+\infty,
\end{gathered}
$$


so that $y e(\lambda) \in \mathfrak{n}_{\tilde{\psi}}$. Furthermore,

$$
\begin{aligned}
& \left\|\eta_{\tilde{\psi}}(y e(\lambda)-y)\right\|^{2}=\psi \circ T\left((y e(\lambda)-y)^{*}(y e(\lambda)-y)\right) \\
& \quad=\psi\left((\mathbf{1}-e(\lambda)) T\left(y^{*} y\right)\right)(\mathbf{1}-e(\lambda)) \\
& \quad=\psi\left(\int_{\lambda}^{\infty} \gamma d e(\gamma)\right) \rightarrow 0 \quad \text { as } \quad \lambda \rightarrow \infty .
\end{aligned}
$$

Similarly, with the spectral decomposition of $T\left(z^{*} z\right)$,

$$
T\left(z^{*} z\right)=\int_{0}^{\infty} \lambda d f(\lambda)
$$

we have $z f(\lambda) \in \mathfrak{n}_{\tilde{\psi}} \cap \mathfrak{n}_{T}$ and

$$
\lim _{\lambda \rightarrow \infty}\left\|\eta_{\tilde{\psi}}(z f(\lambda)-z)\right\|=0 .
$$

By (7), we get

$$
\begin{aligned}
\lim _{\lambda \rightarrow \infty}\left\|\eta_{\tilde{\psi}}\left(y e(\lambda) a^{*}\right)-\eta_{\tilde{\psi}}\left(y a^{*}\right)\right\| & =0, \\
\lim _{\lambda \rightarrow \infty}\left\|\eta_{\tilde{\psi}}(z f(\lambda) b)-\eta_{\tilde{\psi}}(z b)\right\| & =0 .
\end{aligned}
$$

Therefore, we have, by the previous arguments for $x_{0}$,

$$
\begin{aligned}
\tilde{\psi}(a x) & =\left(\eta_{\tilde{\psi}}(z) \mid\left(\eta_{\tilde{\psi}}\left(y a^{*}\right)\right)=\lim _{\lambda \rightarrow \infty}\left(\eta_{\tilde{\psi}}(z f(\lambda)) \mid \eta_{\tilde{\psi}}\left(y e(\lambda) a^{*}\right)\right)\right. \\
& =\lim _{\lambda \rightarrow \infty} \tilde{\psi}\left(a(y e(\lambda))^{*}(z f(\lambda))\right) \\
& \left.=\lim _{\lambda \rightarrow \infty} \tilde{\psi}(y e(\lambda))^{*}(z f(\lambda)) b\right) \\
& =\lim _{\lambda \rightarrow \infty}\left(\eta_{\tilde{\psi}}(z f(\lambda) b) \mid \eta_{\tilde{\psi}}(y e(\lambda))\right) \\
& =\left(\eta_{\psi}(z b) \mid \eta_{\psi}(y)\right)=\tilde{\psi}(x b) .
\end{aligned}
$$

Hence, we may conclude that $(a, b) \in \mathcal{G}\left(\sigma_{-i}^{\tilde{\psi}}\right)$, i.e., $\mathcal{G}\left(\sigma_{-i}^{\psi}\right) \subset \mathcal{G}\left(\sigma_{-i}^{\tilde{\psi}}\right)$.

Now, we know that $x \in \mathcal{M}$ is of exponential type relative to $\left\{\sigma_{t}^{\tilde{\psi}}\right\}$ if and only if

$$
\left\{\begin{array}{l}
x \in \bigcap_{n \in \mathbb{Z}} \mathcal{D}\left(\sigma_{-n i}^{\tilde{\psi}}\right), \\
\sup \left\|\sigma_{-n i}^{\tilde{\psi}}(x)\right\| e^{-c|n|}<+\infty
\end{array}\right.
$$

for some $c>0$. Hence $\mathcal{N}_{\exp }^{\psi} \subset \mathcal{M}_{\exp }^{\tilde{\psi}}$. For each $x \in \mathcal{N}_{\exp }^{\psi}$, we consider

$$
y(\alpha)=\sigma_{\alpha}^{\psi}(x)-\sigma_{\alpha}^{\tilde{\psi}}(x), \quad \alpha \in \mathbb{C} .
$$

The preceding discussion shows that the function $f: \alpha \in \mathbb{C} \mapsto \omega(y(\alpha)) \in \mathbb{C}$ (for any $\left.\omega \in \mathcal{M}_{*}\right)$ is an entire function of exponential type and that $f(-i n)=0, \forall n \in \mathbb{Z}$. Hence $f(\alpha)=0$ for every $\alpha \in \mathbb{C}$, and so we must have $y(\alpha)=0$. This means that

$$
\sigma_{t}^{\psi}(x)=\sigma_{t}^{\tilde{\psi}}(x), \quad x \in \mathcal{N}_{\exp }^{\psi} .
$$

As $\mathcal{N}_{\exp }^{\psi}$ is $\sigma$-weakly dense in $\mathcal{N}$, we conclude (6). 


\section{EXAMPLES}

We now wish to present a few examples in which the action of the operator valued weight can be made explicit. These examples will demonstrate the appropriateness of interpreting the application of an operator valued weight as a (partial) integration. In each of the cases to be discussed, the role of $\mathcal{M}$ will be played by $\mathcal{L}(\mathfrak{H})$ (for an appropriate Hilbert space $\mathfrak{H}$ ), $\tilde{\varphi}$ will be $\operatorname{Tr}$, and $\mathcal{N}$ will be a subalgebra of $\mathcal{M}$ which possesses its own trace $\varphi$. Hence, the condition

$$
\sigma_{t}^{\varphi}(x)=\sigma_{t}^{\tilde{\varphi}}(x), \quad x \in \mathcal{N},
$$

is trivially satisfied. Note also that in the following examples, no distinction will be made between $T$ and its extension $\dot{T}$.

1. Let $G=\mathbb{Z}$, i.e., we are going to consider a discrete, countable abelian group. Take any $x \in \mathcal{L}\left(\ell^{2}(\mathbb{Z})\right)$; we may write $x=\left(x_{i j}\right)_{i, j \in \mathbb{Z}}$. Then (believe it or not), the operator valued weight

$$
T: \mathcal{L}\left(\ell^{2}(\mathbb{Z})\right)_{+} \rightarrow{\widehat{\ell(\mathbb{Z})_{+}}}
$$

is given by

$$
T(x):=\left(x_{n n}\right)_{n \in \mathbb{Z}} .
$$

2. Let $G=\mathbb{T}$, i.e., we are considering the compact, abelian group case. Then the operator valued weight is given by $x \in \mathcal{L}\left(L^{2}(\mathbb{T})\right)_{+} \mapsto \sum_{k \in \mathbb{Z}} u^{k} x u^{-k} \in$ $L^{\infty(\mathbb{T})_{+}}$, where $(u \xi)(\zeta):=\zeta \xi(\zeta)$. Note that if we choose $\epsilon_{i}(\zeta)=\zeta^{i}$, then $\left\{\epsilon_{i}: i \in \mathbb{Z}\right\}$ is a complete, orthonormal system for $L^{2}(\mathbb{T})$. Taking $x=t_{\epsilon_{i}, \epsilon_{j}}$ (here, we define $t_{\epsilon_{i}, \epsilon_{j}} \eta:=\left(\eta \mid \epsilon_{j}\right) \epsilon_{i}$, for any $\left.\eta \in \mathcal{L}\left(L^{2}(\mathbb{T})\right)\right)$, we obtain

$$
\begin{aligned}
(T(x) \xi)(\zeta) & =\sum_{k \in \mathbb{Z}} \hat{\xi}(j-k) \zeta^{i-k} \\
& =\zeta^{i-j} \xi(\zeta)
\end{aligned}
$$

where

$$
\hat{\xi}(n)=\frac{1}{2 \pi} \int_{\mathbb{T}} e^{-i n \zeta} \xi(\zeta) d \zeta .
$$

The previous calculation shows us that, $\forall i, j \in \mathbb{Z}, t_{\epsilon_{i}, \epsilon_{j}} \in \mathfrak{m}_{T}$, so that $T$ is indeed semi-finite. Once again, though, it is necessary to define $T(x)(\omega)$ for any $x \in L^{\infty}(\mathbb{T})_{+}$. Unlike the previous example, however, $T$ is not a conditional expectation, so we cannot hope to define $T(x)(\omega)$ in as elementary a fashion.

We proceed as follows: Let $\mathfrak{H}=L^{2}(\mathbb{T})$; then for any $x \in \mathcal{L}\left(L^{2}(\mathbb{T})\right)_{+}$, we define a map $q_{x}: \mathfrak{H} \rightarrow[0,+\infty]$ via

$$
q_{x}(\xi):=\sum_{k \in \mathbb{Z}}\left\|x^{\frac{1}{2}} u_{-k} \xi\right\| .
$$

$q_{x}$ is then a lower semi-continuous quadratic form, whose domain $\mathcal{D}\left(q_{x}\right)$ includes $\left\{t_{\epsilon_{i}, \epsilon_{i}}: i \in \mathbb{Z}\right\}$ (again, by preceding calculation); hence, to it is associated a positive, self-adjoint operator $H_{x}$. Moreover, due to the form of $q_{x}$, it is clear that $H_{x}$ is affiliated to $\mathcal{N}$. Hence, $\forall \varepsilon>0, H_{x}\left(\mathbf{1}+\varepsilon H_{x}\right)^{-1} \in$ 
$\mathcal{N}_{+}=L^{\infty}(\mathbb{T})_{+}$. Moreover, we know that $\varepsilon_{1} \leq \varepsilon_{2} \rightarrow H_{x}\left(\mathbf{1}+\varepsilon_{2} H_{x}\right)^{-1} \leq$ $H_{x}\left(\mathbf{1}+\varepsilon_{1} H_{x}\right)^{-1}$. We may therefore define

$$
T(x)(\omega):=\lim _{\varepsilon \searrow 0}\left\langle\omega, H_{x}\left(\mathbf{1}+\varepsilon H_{x}\right)^{-1}\right\rangle .
$$

Throughout the remainder of the examples, whenever the action of an operator valued weight $T$ is exhibited as a summation (or integration), it is to be understood in the above context, viz., we should consider the quadratic form induced by our definition of $T$ in a way entirely analogous to (8), and then consider the appropriate (possibly unbounded) operator.

3. Let $G=\mathbb{R}$, i.e., we are considering a separable, locally compact but noncompact, abelian group. In this case, the operator valued weight is given by

$$
T: x \in \mathcal{L}\left(L^{2}(\mathbb{R})\right)_{+} \mapsto \int_{\widehat{\mathbb{R}}=\mathbb{R}} u(\lambda) x u(-\lambda) d \lambda \in \widehat{L^{\infty(\mathbb{R})}}+
$$

where

$$
(u(\lambda) \xi)(s):=e^{i \lambda s} \xi(s), \quad \forall \xi \in L^{2}(\mathbb{R})
$$

Taking $\left\{\epsilon_{i}: \epsilon_{i} \in L^{2}(\mathbb{R}) \cap L^{\infty}(\mathbb{R}), i \in \mathbb{N}\right\}$, a complete orthonormal system for $L^{2}(\mathbb{R})$, and again taking $x$ to be $t_{\epsilon_{i}, \epsilon_{j}}$, we obtain

$$
(T(x) \xi)(s)=\epsilon_{i}(s) \overline{\epsilon_{j}(s)} \xi(s), \quad x=t_{\epsilon_{i}, \epsilon_{j}} .
$$

In the general setting, where $G$ is any (separable) locally compact, abelian group, with dual group $\widehat{G}$, we will have $T: \mathcal{L}\left(L^{2}(G)\right)_{+} \rightarrow \widehat{L^{\infty(G)}}+$ given by

$$
T(x):=\int_{\widehat{G}} u(p) x u(-p) d p
$$

where

$$
(u(p) \xi)(g):=\langle p, g\rangle \xi(g), \quad \forall \xi \in L^{2}(G)
$$

Here, of course, $\langle\cdot, \cdot\rangle$ denotes the duality between $\widehat{G}$ and $G$.

1. Suppose $G$ is a discrete, separable group, but not necessarily abelian. If $G$ is ICC (i.e., infinite conjugacy class), then $\mathcal{R}_{\ell}(G) \subset \mathcal{L}\left(\ell^{2}(G)\right)$ is a $\mathrm{II}_{1}$-factor, and therefore possesses a tracial state $\tau$. So again, we are motivated to look for an operator valued weight $T: \mathcal{L}\left(\ell^{2}(G)\right)_{+} \rightarrow \widehat{\mathcal{R}_{\ell}(G)_{+}}$.

In this setting, the operator valued weight is given by

$$
T(x):=\sum_{g \in G} \rho(g) x \rho(g)^{*},
$$

where

$$
\rho(g) \xi(h):=\xi(h g), \quad \forall \xi \in \ell^{2}(G) .
$$

To see that this is so, we note immediately that $T$ is clearly positive homogeneous and linear on all of $\mathcal{L}\left(\ell^{2}(G)\right)_{+}$. If we define $\epsilon_{g}: G \rightarrow \mathbb{C}, g \in G$, via $\epsilon_{g}(s):=\delta_{s, g}$, then clearly $\left\{\epsilon_{g}: g \in G\right\}$ is a complete, orthonormal system for 
$\ell^{2}(G)$. Take $x=t_{\epsilon_{g}, \epsilon_{h}}$; then $x \in \mathcal{L} \mathcal{F}\left(\ell^{2}(G)\right)$ (the set of finite rank operators); we calculate

$$
\begin{aligned}
T(x)=T\left(t_{\epsilon_{g}, \epsilon_{h}}\right) & =\sum_{s \in G} \rho(s) t_{\epsilon_{g}, \epsilon_{h}} \rho(s)^{*}, \\
& =\sum_{s \in G} \rho(s) t_{\epsilon_{g}, \epsilon_{h}} \rho\left(s^{-1}\right)=\sum_{s \in G} \rho(s) t_{\epsilon_{g}, \epsilon_{h s^{-1}}} \\
& =\sum_{s \in G} t_{\epsilon_{g s^{-1}, \epsilon_{h-1}} .}
\end{aligned}
$$

Now, we will demonstrate that $T\left(t_{\epsilon_{g}, \epsilon_{h}}\right)$ is actually equal to $\lambda\left(g h^{-1}\right)$, where (as usual)

$$
\lambda(g) \xi(h):=\xi\left(g^{-1} h\right), \quad \forall \xi \in \ell^{2}(G) .
$$

For any $k \in G$, we compute

$$
\begin{aligned}
T\left(t_{\epsilon_{g}, \epsilon_{h}}\right) \epsilon_{k} & =\sum_{s \in G} t_{\epsilon_{g s^{-1}}, \epsilon_{h s^{-1}}} \epsilon_{k}=\sum_{s \in G}\left(\epsilon_{k} \mid \epsilon_{h s^{-1}}\right) \epsilon_{g s^{-1}} \\
& =\sum_{s \in G} \delta_{k, h s^{-1}} \epsilon_{g s^{-1}}=\sum_{s \in G} \delta_{s, k^{-1} h} \epsilon_{g s^{-1}} \\
& =\epsilon_{g h^{-1} k}=\lambda\left(g h^{-1}\right) \epsilon_{k} .
\end{aligned}
$$

Hence, we see that $T\left(\left\{t_{\epsilon_{g}, \epsilon_{h}}: g, h \in G\right\}\right) \subset \mathcal{R}_{\ell}(G)$; thus $T$ is semi-finite. Also, $T$ is faithful: to say $T\left(x^{*} x\right)=0$ implies $\sum_{s \in G} \rho(s) x^{*} x \rho(s)^{*}=0$. But $\rho(s) x^{*} x \rho(s)^{*}=\left(x \rho(s)^{*}\right)^{*}\left(x \rho(s)^{*}\right)$, so $T\left(x^{*} x\right)$ is a sum of positive elements, which can only be 0 if each $\left(x \rho(s)^{*}\right)^{*}\left(x \rho(s)^{*}\right)=0$. But since each $\rho(s)$ is unitary, this will only be so if $x^{*} x=x=0$. Finally, the form of $T$ ensures its normality.

It is also interesting to note that the form of $T$ is that of "integration over the commutant;" specifically, we are summing over $\{\rho(s): s \in G\}$, and we recognize that $\{\rho(s): s \in G\}^{\prime \prime}=\mathcal{R}_{r}(G) \cong \mathcal{R}_{\ell}(G)^{\prime}$. This lends credence to our earlier claim that the application of an operator valued weight should be interpreted as integration.

As an aside, we mention that, on $\mathfrak{m}_{T}, T$ has the form

$$
T(x)=\sum_{g \in G} \operatorname{Tr}\left(\lambda(g)^{*} x\right) \lambda(g) .
$$

Expressing $T$ via the above allows us to view the application of $T$ as a kind of "Fourier transform."

2. Let $A_{n}=M_{2}(\mathbb{C}) \otimes \cdots \otimes M_{2}(\mathbb{C}) \cong M_{2^{n}}(\mathbb{C}), \mathcal{A}_{0}=\underline{\lim } A_{n}$, the algebraic direct limit of the $A_{n}$, and $\mathcal{A}=\widehat{\mathcal{A}_{0}}$, the $C^{*}$-algebraic direct limit of the $A_{n}$. Note that in this context we may (and shall) consider $\mathcal{A}$ to be the " $C^{*}$-algebraic infinite tensor product" $\quad M_{2}(\mathbb{C}) \otimes M_{2}(\mathbb{C}) \otimes \cdots$. Now, each $A_{n}$ possesses a tracial state $\tau_{n}:=2^{-n} \operatorname{Tr}_{n}$, where $\operatorname{Tr}_{n}$ denotes the usual trace on $M_{2^{n}}(\mathbb{C})$. These, in turn, induce a tracial state on $\mathcal{A}$, which we shall call $\tau$.

In the usual way (i.e., via the GNS construction), we obtain a representation of $\mathcal{A}$, viz., $\left\{\pi_{\tau}, \mathfrak{H}_{\tau}, \xi_{\tau}\right\}$. Then, we know that $\pi_{\tau}(\mathcal{A})^{\prime \prime} \subset \mathcal{L}\left(\mathfrak{H}_{\tau}\right)$ is a $\mathrm{II}_{1}$-factor - in fact, it is the AFD $\mathrm{II}_{1}$-factor $\mathcal{R}_{0}$. The trace on $\mathcal{R}_{0}$, which will also be denoted by $\tau$, is given by $\omega_{\xi_{\tau}}$. Hence, we are now looking for the operator valued weight $T: \mathcal{L}\left(\mathfrak{H}_{\tau}\right)_{+} \rightarrow \widehat{\mathcal{R}_{0+}}$ which satisfies $\operatorname{Tr}=\tau \circ T$. 
To find $T$, it is helpful to change perspective. Let's start by defining $\widetilde{\mathfrak{H}}$ to be $\left\{M_{2}(\mathbb{C}), \frac{1}{2} \operatorname{Tr}_{1}\right\}$, i.e., $M_{2}(\mathbb{C})$ considered as a Hilbert space, via the inner product $(X \mid Y):=\operatorname{Tr}_{1}\left(Y^{*} X\right)$. We next define $\mathfrak{H}_{n}$ via $\mathfrak{H}_{n}:=\widetilde{\mathfrak{H}} \otimes \cdots \otimes \widetilde{\mathfrak{H}}(n$ times). We embed $\mathfrak{H}_{n} \hookrightarrow \mathfrak{H}_{n+1}$ via $\xi \mapsto \xi \otimes \mathbf{1}_{1}$, where $\mathbf{1}_{1}$ is the identity matrix in $M_{2}(\mathbb{C})$. Since this map is clearly an isometry, we are free to consider the "Hilbert space infinite tensor product;" we shall call this $\mathfrak{H}$.

Now, it is easy to see that $\mathcal{A}_{0}$ acts on $\mathfrak{H}$ in a natural way via both left and right multiplication; moreover, this action is bounded. Thus, we may consider $\lambda_{\ell}\left(\mathcal{A}_{0}\right), \lambda_{r}\left(\mathcal{A}_{0}\right) \subset \mathcal{L}(\mathfrak{H})$. It is also not difficult to see that we actually have

$$
\mathfrak{H} \cong \mathfrak{H}_{\tau} \quad \text { and } \quad \lambda_{\ell}\left(\mathcal{A}_{0}\right)^{\prime \prime} \cong \mathcal{R}_{0} .
$$

Let's contemplate what occurs at the $n^{\text {th }}$ "stage" of the direct limiting process. We have $A_{n}$ acting on $\mathfrak{H}_{n}$ from both the left and right; in fact, $\mathcal{L}\left(\mathfrak{H}_{n}\right) \cong A_{2 n}=A_{n} \otimes A_{n}$. Note also that we have $\operatorname{Tr}_{2 n}=\operatorname{Tr}_{n} \otimes \operatorname{Tr}_{n}$, and $\tau_{2 n}=\tau_{n} \otimes \tau_{n}$. We can obtain a conditional expectation $\mathcal{E}_{n}: \mathcal{L}\left(\mathfrak{H}_{n}\right) \rightarrow A_{n}$ via

$$
\mathcal{E}_{n}(x):=\int_{\mathcal{U}\left(A_{n}^{\prime}\right)} u x u^{*} d u, \quad \forall x \in \mathcal{L}\left(\mathfrak{H}_{n}\right) .
$$

Here, we are integrating with respect to the normalized Haar measure over the compact group $\mathcal{U}\left(A_{n}^{\prime}\right) \cong \mathrm{U}\left(2^{n}\right)$. It is clear that we have $\tau_{2 n}=\tau_{n} \circ \mathcal{E}_{n}$. However, note that if we want to have $T_{n}: \mathcal{L}\left(\mathfrak{H}_{n}\right) \rightarrow A_{n}$ such that $\operatorname{Tr}_{2 n}=$ $\tau_{n} \circ T_{n}$, we must define $T_{n}:=2^{2 n} \mathcal{E}_{n}$.

These observations guide us to the proper formulation of $T$. For any $x \in$ $\mathcal{L}\left(\mathfrak{H}_{\tau}\right)_{+}$, we define

$$
T(x):=\lim _{n \rightarrow \infty} 2^{2 n} \int_{\mathcal{U}\left(A_{n}\right)} \operatorname{Ad}\left(\mathrm{J}_{\tau} u \mathrm{~J}_{\tau}\right) x d u,
$$

where $\mathrm{J}_{\tau}$ is the modular conjugation. (As $\tau$ is a trace, we have $\mathrm{J}_{\tau} \eta_{\tau}(y)=$ $\eta_{\tau}\left(y^{*}\right)$, with $\eta_{\tau}: \mathcal{A} \rightarrow \mathfrak{H}_{\tau}$.) For any $x \in \mathcal{A}_{0}$, our previous considerations show that this defintion yields the desired results; as $\mathcal{A}_{0}$ is $\sigma$-weakly dense in $\mathcal{L}\left(\mathfrak{H}_{\tau}\right)$, we observe (once again by an appeal to normality) that $T$ is the correct operator valued weight.

It is also interesting to note that it is possible to consider a different limiting process applied to the previously defined $\mathcal{E}_{n}$ 's. As each $\mathcal{E}_{n}$ is a projection of norm one, for any $x \in \mathcal{L}(\mathfrak{H}),\left\{\left(\tau \circ \mathcal{E}_{n}\right)(x): n \in \mathbb{N}\right\} \in \ell^{\infty}(\mathbb{N})$; if we choose $\omega$ a free ultrafilter (i.e., $\omega \in \beta \mathbb{N} \backslash \mathbb{N}$, where, as usual, $\beta \mathbb{N}$ is the Stone-Čech compactification of $\mathbb{N}$ ), we may define

$$
\rho_{\omega}(x):=\lim _{n \rightarrow \omega}\left(\tau \circ \mathcal{E}_{n}\right)(x) .
$$

Then $\rho_{\omega}$ is a hypertrace on $\mathcal{L}(\mathfrak{H})$. It is, however, far from normal; it is singular [Tak2] $-\rho_{\omega}$ is a transcendental object. Similarly, if we consider, for any $x \in \mathcal{L}(\mathfrak{H}),\left\{\mathcal{E}_{n}(x): n \in \mathbb{N}\right\} \in \ell^{\infty}(\mathbb{N}, \mathcal{L}(\mathfrak{H}))$, we may define

$$
\Phi_{\omega}(x):=\lim _{n \rightarrow \omega} \mathcal{E}_{n}(x) .
$$

$\Phi_{\omega}: \mathcal{L}(\mathfrak{H}) \rightarrow \mathcal{R}_{0}$ is a conditional expectation, but it too is highly singular. So, it seems, we are faced with a choice: to forego boundedness for the sake of normality (i.e., work with $T$ ), or retain boundedness and give up $\sigma$-weak continuity. Both techniques have utility; circumstances will dictate which option is more useful. 


\section{Conclusions}

The above proof demonstrates that it is possible to arrive at a complete theory of operator valued weights without recourse to structure theory. This is very satisfying if one adopts the point of view that integration theory is fundamental to further study in the theory of von Neumann algebras, and that structure theory should be a "consequence" of the theory of non-commutative integration.

\section{REFERENCES}

[Haa1] U. Haagerup, Operator Valued Weights in von Neumann Algebras, I, J. Func. Anal. 32 175-206 (1979). MR 81e:46049a

[Haa2] U. Haagerup, Operator Valued Weights in von Neumann Algebras, II, J. Func. Anal. 33 339-361 (1979). MR 81e:46049b

[Hir] M. Hirakawa, A Generalization of $\varphi$-conditional Expectation and Operator Valued Weight, Publ. Res. Inst. Math. Sci. 28 289-297 (1992). MR 93a:46121

[Mas] T. Masuda, A Note on a Theorem of A. Connes on Radon-Nikodym Cocycles, Publ. Res. Inst. Math. Sci. 20 131-136 (1984). MR 85g:46081

[Tak1] M. Takesaki, Conditional Expectations in von Neumann Algebras, J. Func. Anal., 9 306320 (1972). MR 46:2445

[Tak2] M. Takesaki, Theory of Operator Algebras I, Springer-Verlag, Chapter III §2 (1979). MR 81e: 46038

[Tak3] M. Takesaki, Theory of Operator Algebras II, Chapter VIII §3; to appear.

Department of Mathematics, University of California, los Angeles, Los Angeles, CALIFORNIA 90095-1555

Current address: Department of Mathematics, Illinois State University, Normal, Illinois 617904520

E-mail address: afalcone@math.ilstu.edu

Department of Mathematics, University of California, los Angeles, Los Angeles, CALIFORnia 90095-1555

E-mail address: mt@math.ucla.edu 